\title{
NMSSM with a singlino LSP: possible challenges for searches for supersymmetry at the LHC
}

\author{
Ulrich Ellwanger ${ }^{a, b}$ and Ana M. Teixeira ${ }^{c}$ \\ ${ }^{a}$ LPT, UMR 8627, CNRS, Université de Paris-Sud, \\ 91405 Orsay, France \\ ${ }^{b}$ School of Physics and Astronomy, University of Southampton, \\ Highfield, Southampton SO17 1BJ, U.K. \\ ${ }^{c}$ Laboratoire de Physique Corpusculaire, CNRS/IN2P3 - UMR 6533 \\ Campus des Cézeaux, 24 Av. des Landais, F-63171 Aubière, France \\ E-mail: Ulrich.Ellwanger@th.u-psud.fr, Ana.Teixeira@clermont.in2p3.fr
}

ABSTRACT: A light singlino in the NMSSM can reduce considerably the missing transverse energy at the end of sparticle decay cascades; instead, light NMSSM-specific Higgs bosons can be produced. Such scenarios can be consistent with present constraints from the LHC with all sparticle masses below $\sim 1 \mathrm{TeV}$. We discuss search strategies, which do not rely on missing transverse energy, for such scenarios at the next run of the LHC near $14 \mathrm{TeV}$.

KEYWords: Supersymmetry Phenomenology

ARXIV EPRINT: 1406.7221 


\section{Contents}

1 Introduction 1

2 "Missing" missing energy in the NMSSM 3

3 Towards the extraction of signals in $b \bar{b}+\tau^{+} \tau^{-}$final states at $14 \mathrm{TeV} \quad 9$

4 Conclusions and outlook $\quad 15$

\section{Introduction}

One of the main tasks of the LHC was and will be the search for supersymmetric (SUSY) particles. The largest production cross sections are expected for gluinos $(\tilde{g})$ and squarks $(\tilde{q})$ of the first generation. After the first run of the LHC at a center of mass (c.m.) energy of mostly $8 \mathrm{TeV}$, no significant excesses have been observed in corresponding search channels $[1,2]$ (see [3] for a recent summary).

The absence of excess events can be interpreted in terms of lower bounds on gluino and squark masses, once assumptions on their decay cascades are made. These depend on the masses and couplings of many other SUSY particles, at least on the mass of the lightest SUSY particle (LSP). Within simplified models (assuming simple 1-step decay cascades) or the Minimal SUSY extension of the Standard Model (MSSM), lower bounds on gluino and squark masses are typically in the $1.2-2 \mathrm{TeV}$ range, and $\sim 1.7 \mathrm{TeV}$ if gluino and squark masses are assumed to be similar [1,2]. Although these constraints do not rule out the MSSM, they eliminate a significant part of its "natural" parameter space [4].

However, the MSSM is not the only SUSY extension of the Standard Model (SM) which alleviates the hierarchy problem, provides an acceptable dark matter candidate and leads to Grand Unification of the running gauge couplings. In the present paper we consider the Next-to-Minimal SUSY extension of the Standard Model (NMSSM) [5], where the coupling of the two Higgs doublets of the MSSM to an additional gauge singlet field $S$ renders more natural a value of $\sim 126 \mathrm{GeV}$ of the SM-like Higgs boson [6-11], while preserving the attractive features of the MSSM. Besides the Higgs sector, the NMSSM differs from the MSSM through the presence of an additional neutralino (the singlino, the fermionic component of the singlet superfield). The singlino can be the LSP, which can modify considerably the SUSY particle decay cascades [12-20].

The strongest constraints from searches for gluinos and squarks of the first generation originate from channels where one looks for events with jets with large transverse momentum $p_{T}$ and missing transverse energy $E_{T}^{\text {miss }}[1,2]$. The $E_{T}^{\text {miss }}$ is due to having all SUSY decay cascades ending in a stable LSP (under the assumption of R-parity conservation), which escapes detection (if neutral, as required for dark matter). 
In the present paper we point out that a singlino-like LSP in the NMSSM can reduce significantly the missing transverse energy at the end of SUSY particle decay cascades. This is due to the kinematics of the last process in a SUSY particle decay chain, NLSP $\rightarrow$ LSP $+X$, where NLSP denotes the Next-to-LSP, and $X$ a particle (e.g. a Higgs boson) decaying into visible components of the SM. For a light LSP, if the mass of $X$ is close to the mass of the NLSP, little energy and momentum are transferred from the NLSP to the LSP; most of the energy is transferred to $X$. Correspondingly, the LSP in the final state leads to little $E_{T}^{\text {miss }}$, whereas large $E_{T}^{\text {miss }}$ is one of the relevant search criteria for SUSY particles in general.

The possibility to discover squarks and gluinos without relying on $E_{T}^{\text {miss }}$, but on leptons, has been studied earlier in [21-23]. The study of [23] discusses decays of an NLSP into a scalar (decaying visibly into SM particles) and the LSP, referring to the NMSSM without, however, considering the particular kinematic configurations analysed below.

A scenario similar to the one discussed here has been named "Stealth Supersymmetry" $[24,25]$. There, however, a complete "stealth sector" is added to the MSSM in order to obtain the above kinematic configuration of the NLSP decay.

An extensive survey of present constraints on gluinos from searches, including several without relying on $E_{T}^{\text {miss }}$, is given in [26]. Among the scenarios analysed in [26] are socalled "minimal Hidden Valley" models. These are similar to the ones considered here after replacing the extra singlet scalar and its fermionic superpartner [26] by the corresponding states of the NMSSM (and the NLSP higgsino by a bino-like NLSP). It was already found in [26] that the kinematic configuration discussed above leads to the weakest constraints.

The present scenario is opposite to the one of "compressed supersymmetry" [27-32] where the masses of the NLSP and the LSP are assumed to be similar, and little energy is transferred to $X$. Then jets (or leptons) with large $p_{T}$ would be rare. Moreover, unless a hard jet is emitted from the initial state ("monojet"), the $E_{T}^{\text {miss }}$ due to two LSPs emitted from two SUSY particles back-to-back in the transverse plane tend to cancel.

In the MSSM, the kinematic configuration considered here cannot play a major rôle: a light LSP (with a mass of a few $\mathrm{GeV}$ ) can only be bino-like, since winos or higgsinos would have charged partners with similar masses, already ruled out by LEP (see [33] and references therein). All squarks - appearing also in gluino decays - have hypercharge and hence couple to the bino. If the LSP is a very light bino, squarks will in general decay directly into the bino, and hardly pass through an NLSP (e.g. a heavier neutralino or chargino) and a state $X$ (a Higgs, $Z$ or $W$ boson). Thus only a fraction of cascade decays leads to a reduction of $E_{T}^{\text {miss }}$, so that the interpretation of the absence of signal events in terms of lower bounds on SUSY particle masses remains practically unchanged.

On the other hand, in the NMSSM the bino can be the NLSP, the singlino a light LSP, and $X$ a priori a Higgs, a $Z$ or even a $W$ boson (if the NLSP is a chargino). Then the decays of $X$ can still give rise to missing energy in the form of neutrinos; this is the case for the decays of the $W$ and $Z$ bosons, and also for the SM-like Higgs (when it decays via $W W^{*}$ or $\left.Z Z^{*}\right)$.

However, in the NMSSM additional Higgs bosons exist, which can be lighter than the $Z$ boson and are not excluded by LEP due to small couplings to $Z Z$ (see $[5,34]$ and 
references therein). A lighter CP-even Higgs boson $H_{1}$ with a mass below $M_{Z}$ would have very small decay rates into $W W^{*}$, but decay dominantly into $b \bar{b}$ and, to some extent, into $\tau^{+} \tau^{-}$. Although the latter decays can also give rise to some $E_{T}^{\text {miss }}$, the scenario NLSP $\rightarrow$ LSP $+H_{1}$ with $M_{H_{1}} \lesssim M_{\mathrm{NLSP}}<M_{Z}$ would be the most difficult one with respect to signatures based on $E_{T}^{\text {miss }}$. (Subsequently we denote scenarios with as little $E_{T}^{\text {miss }}$ as possible as "worst case".)

In the case of squark/gluino pair production, some $E_{T}^{\text {miss }}$ can also originate from $W$, $Z$ and/or Higgs decays which appear during decay cascades involving charginos and/or heavier neutralinos. Again, a "worst case" scenario would be one where this does not happen if, for instance, the chargino and heavier neutralino masses are close to (or above) the squark masses.

In the present paper we concentrate on such "worst case" scenarios: first, we present the properties of points in the NMSSM which are not excluded by present SUSY searches although all sparticle masses are below $\sim 1 \mathrm{TeV}$. Second, we propose search strategies for these difficult scenarios, putting forward an analysis of events at the LHC near $14 \mathrm{TeV}$ c.m. energy, based on the decay products of two $H_{1}$ bosons in the $b \bar{b} \tau^{+} \tau^{-}+$jets final state. Our simulations indicate that, for not excessively heavy squarks and gluinos (i.e. a not too small production cross section), a signal can be visible above Standard Model backgrounds.

In the next section we discuss in detail scenarios within the general NMSSM, in which $E_{T}^{\text {miss }}$ is reduced for kinematic reasons. Results of event simulations of such a benchmark point are discussed, which explain the reduced sensitivity of present SUSY searches to such a scenario. We also discuss simplified models with varying LSP and $H_{1}$ masses, and the corresponding reduction of signal events. In section 3 we attempt to extract signals for $H_{1}$ pair production at the LHC with $14 \mathrm{TeV}$ c.m. energy, with dedicated cuts which do not rely on $E_{T}^{\text {miss }}$. Instead, we attempt to identify $b$-jets and $\tau$-leptons from boosted $H_{1}$ bosons with the help of a jet reconstruction with a small jet cone radius $R=0.15$. Section 4 contains a summary and conclusions.

\section{2 "Missing" missing energy in the NMSSM}

Given a possible last step in a SUSY particle decay chain NLSP $\rightarrow$ LSP $+X$ in the limit of a narrow phase space, $M_{\mathrm{NLSP}}-\left(M_{\mathrm{LSP}}+M_{X}\right) \ll M_{\mathrm{NLSP}}$, the energy (momentum) transferred from the NLSP to the LSP in the laboratory frame is proportional to the ratio of masses:

$$
\frac{E_{\mathrm{LSP}}}{E_{\mathrm{NLSP}}} \simeq \frac{M_{\mathrm{LSP}}}{M_{\mathrm{NLSP}}}
$$

Hence, if the LSP is light, little (missing transverse) energy is transferred to the LSP; the transverse energy is carried away by $X$. The effect is the more important the narrower the phase space is. As explained in the Introduction, such a scenario is difficult to realise in the MSSM where such a light LSP must be bino-like.

The particle content of the NMSSM differs from that of the MSSM by an additional singlino-like neutralino $\tilde{S}$, and additional singlet-like CP-even and CP-odd Higgs bosons [5]. Notably the NMSSM spectrum contains three CP-even Higgs bosons $H_{i}, i=1,2,3$ (ordered 
in mass). The singlino-like neutralino can be the LSP with a bino-like NLSP (as occurs for the regions of parameter space considered below). Then the above scenario of little $E_{T}^{\text {miss }}$ being transferred to the LSP can be realised with a singlet-like CP-even Higgs boson $H_{1}$ playing the rôle of $X$, whose subsequent decays give rise to little invisible transverse energy in the form of neutrinos. Typical values for the masses would be a few $\mathrm{GeV}$ for the

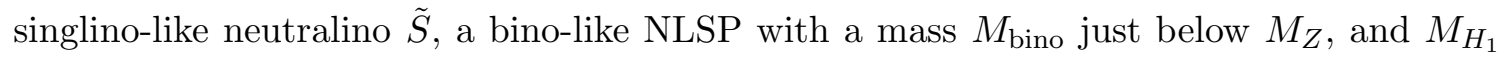
just below $M_{\text {bino }}-M_{\tilde{S}}$. Note that, due to its reduced coupling to the $Z$ boson, such a light $H_{1}$ can still be compatible with constraints from Higgs searches at LEP [34].

In the simplest $\mathbb{Z}_{3}$ invariant realisation of the NMSSM, the diagonal elements of the mass matrices for the (pure) singlet-like states $\tilde{S}, H_{S}$ and $A_{S}$ satisfy [19]

$$
M_{\tilde{S}}^{2} \sim M_{H_{S}}^{2}+\frac{1}{3} M_{A_{S}}^{2},
$$

which forbids $M_{H_{S}} \gg M_{\tilde{S}}$ and hence $M_{H_{1}} \gg M_{\mathrm{LSP}}$ unless soft SUSY breaking trilinear couplings are in the multi- $\mathrm{TeV}$ range, in which case there can be strong deviations from the equality of eq. (2.2) for the mass eigenstates (after diagonalization of the mass matrices).

However, $M_{H_{S}} \gg M_{\tilde{S}}$ is possible in the presence of $\mathbb{Z}_{3}$ violating terms like a soft SUSY breaking tadpole term $\xi_{S} S$, and/or a holomorphic soft SUSY breaking mass term $\frac{1}{2} m_{S}^{\prime 2} S^{2}+$ h. c.. Such terms are generated automatically in gauge mediated supersymmetry breaking (GMSB), if the singlet superfield has couplings to the messenger fields [35].

Hence we consider in the following a general NMSSM, still with a $\mathbb{Z}_{3}$ invariant superpotential

$$
W_{\mathrm{NMSSM}}=\lambda \hat{S} \hat{H}_{u} \cdot \hat{H}_{d}+\frac{\kappa}{3} \hat{S}^{3}+\ldots
$$

In the above hatted letters denote superfields, and the ellipses denote the MSSM-like Yukawa couplings of $\hat{H}_{u}$ and $\hat{H}_{d}$ to the quark and lepton superfields. We allow for the following NMSSM specific soft SUSY breaking terms

$$
-\mathcal{L}_{\mathrm{NMSSM}}^{\text {soft }}=m_{S}^{2}|S|^{2}+\left(\lambda A_{\lambda} H_{u} H_{d} S+\xi_{S} S+\frac{1}{2} m_{S}^{\prime 2} S^{2}+\frac{1}{3} \kappa A_{\kappa} S^{3}\right)+\text { h. c. . }
$$

The remaining MSSM-like soft breaking terms are chosen to be flavour and CP conserving; the first two sfermion families are degenerate, and LR-mixing is only taken into account for the third generation of squarks and leptons.

As can be seen from eq. (2.3), a vacuum expectation value $\langle S\rangle$ generates an effective $\mu_{\text {eff }}$ term $\mu_{\text {eff }}=\lambda\langle S\rangle$, which has to be larger than $\sim 100 \mathrm{GeV}$ for the charged higgsinos to satisfy bounds from LEP. Given the diagonal singlino mass term $M_{\tilde{S}}=2 \kappa\langle S\rangle$, a singlino mass of a few $\mathrm{GeV}$ is obtained for $\kappa$ about two orders of magnitude smaller than $\lambda$.

For completeness we comment on the possibilities to obtain consistent properties of dark matter in such a scenario. Within GMSB, a gravitino can be lighter than $\tilde{S}$ which would thus not be the "true" LSP, but decay radiatively into a gravitino and a photon (through a small photino component from a non-vanishing mixing with the bino/wino). However, the singlino life time would be so large that this decay would occur outside the detectors and have no impact on our subsequent analyses. (On the other hand, the singlino life time should not exceed $\sim 100 \mathrm{~s}$ in order not to spoil nucleosynthesis unless the NLSP 


\begin{tabular}{|c|c|c|c|c|c|}
\hline Parameter & Value & Parameter & Value & Particle(s) & Mass \\
\hline$\lambda$ & $6.5 \times 10^{-3}$ & $M_{1}$ & $90 \mathrm{GeV}$ & $M_{H_{1}}$ & $83 \mathrm{GeV}$ \\
\hline$\kappa$ & $1.9 \times 10^{-5}$ & $M_{2}$ & $950 \mathrm{GeV}$ & $M_{H_{2}}$ & $123.2 \mathrm{GeV}$ \\
\hline $\tan \beta$ & 20 & $M_{3}$ & $830 \mathrm{GeV}$ & $M_{H_{3}, A_{2}, H^{ \pm}}$ & $\sim 950 \mathrm{GeV}$ \\
\hline$\mu_{\text {eff }}$ & $900 \mathrm{GeV}$ & $A_{t}$ & $-1500 \mathrm{GeV}$ & $M_{A_{1}}$ & $12.9 \mathrm{GeV}$ \\
\hline$\xi_{S}$ & $-1.02 \times 10^{9} \mathrm{GeV}^{3}$ & $A_{b}$ & $-1000 \mathrm{GeV}$ & $M_{\text {squarks }}$ & $\sim 860 \mathrm{GeV}$ \\
\hline$m_{S}^{\prime}{ }^{2}$ & $3.6 \times 10^{3} \mathrm{GeV}^{2}$ & $m_{\text {sleptons }}$ & $600 \mathrm{GeV}$ & $M_{\text {stop1 }}$ & $810 \mathrm{GeV}$ \\
\hline$A_{\kappa}$ & $0 \mathrm{GeV}$ & $m_{\text {squarks }}(\mathrm{u}, \mathrm{d}, \mathrm{s}, \mathrm{c})$ & $830 \mathrm{GeV}$ & $M_{\text {stop} 2}$ & $1060 \mathrm{GeV}$ \\
\hline$A_{\lambda}$ & $50 \mathrm{GeV}$ & $m_{\text {squarks }}(\mathrm{t}, \mathrm{b})$ & $900 \mathrm{GeV}$ & $M_{\text {gluino }}$ & $893 \mathrm{GeV}$ \\
\hline & & & & $M_{\chi_{1}^{0}}$ & $5.26 \mathrm{GeV}$ \\
\hline & & & & $M_{\chi_{2}^{0}}$ & $89 \mathrm{GeV}$ \\
\hline
\end{tabular}

Table 1. Parameters (left and middle column) and particle masses (right column) of a NMSSM benchmark point.

density is diluted through entropy production.) Alternatively, the singlino relic density can be reduced to comply with the observed dark matter relic density through the exchange of a CP-odd Higgs state $A_{S}$ in the s-channel, provided $M_{A_{S}} \sim 2 M_{\tilde{S}}$. We have checked that this is indeed possible, and the benchmark point given below has this property.

Returning to the issue of $E_{T}^{\text {miss }}$, its suppression is maximised if no neutrinos from $Z / W$ decays are emitted during squark/gluino decay cascades. In a truly "worst case scenario" winos, higgsinos, sleptons, stops and sbottoms are not produced neither in squark nor in gluino decays. Bino $\rightarrow Z+$ singlino decays are impossible, if the bino mass is below $M_{Z}$. In table 1 we give the parameters and particle masses of a benchmark point with these properties, for which physical masses and decay branching fractions have been obtained with the public code NMSSMTools_4.2.1 [36, 37].

The parameters $M_{1}, M_{2}, M_{3} A_{t}, A_{b}$ in table 1 denote the soft SUSY breaking bino-, wino- and gluino mass terms and Higgs-stop, Higgs-sbottom trilinear couplings, respectively. The lightest neutralinos are denoted by $\chi_{1}^{0}$ (singlino-like) and $\chi_{2}^{0}$ (bino-like), respectively. The gaugino mass terms are non-universal, but lead to a go-theorem for branching fractions corresponding to a simplified model: squarks decay to $100 \%$ into $\chi_{2}^{0}$ and the corresponding quark, $\chi_{2}^{0}$ to $100 \%$ into $\chi_{1}^{0}+H_{1}$. Gluinos decay with approximately equal branching fractions only into squarks + quarks of the first two generations. Hence the decay chains are

$$
\begin{aligned}
& \tilde{q} \rightarrow q \chi_{2}^{0} \rightarrow q H_{1} \chi_{1}^{0} ; \\
& \tilde{g} \rightarrow \tilde{q} q .
\end{aligned}
$$

The small value of $\lambda$ suppresses mixings of the singlet-like $A_{1}$ with the doublet-like $A_{2}$ such that $\chi_{2}^{0}$ decays into $\chi_{1}^{0}+H_{1}$ in spite of the lighter $A_{1}$, and suppresses mixings of the singlet-like $\chi_{1}^{0}$ with higgsinos/winos which can lead to unacceptable invisible decay rates of the Standard Model-like $H_{2}$ into $\chi_{1}^{0}+\chi_{1}^{0}$. Despite the small value of $\lambda$ (but due to the large value of $\tan \beta$ ) the mass of $H_{2}-$ still consistent with $\sim 126 \mathrm{GeV}$ within the expected 
theoretical error in NMSSMTools - is larger than in the MSSM due to mixing of $\mathrm{H}_{2}$ with $H_{1}[38]$.

Despite the large value of $\tan \beta$, constraints from flavour physics (notably $B$ decays, see [39]) are satisfied: first, the charged Higgs boson as well as squark, gluino and chargino masses are in the $860-1000 \mathrm{GeV}$ range (and $A_{t}$ negative, but $\left|A_{t}\right|$ not too large), hence "MSSM"-like contributions to $\varepsilon_{K}, B_{s, d}-\bar{B}_{s, d}$ mixing and $b \rightarrow s \gamma$ are not excessively large. Contributions from CP-odd scalars to $B_{s, d} \rightarrow \mu^{+} \mu^{-}$are small since the MSSM-like state $A_{2}$ is also heavy, whereas the light NMSSM-like state $A_{1}$ is practically decoupled. Thus all phenomenological constraints (except for the muon anomalous magnetic moment) tested in NMSSMTools are satisfied. (The public code SUSY_FLAVOR v2.11 [40, 41] was used to numerically evaluate the contributions to $K$-mixing observables, notably to $\varepsilon_{K}$.)

Due to the small width of $A_{1}$ it is difficult, however, to determine the dark matter relic density accurately with the code micrOMEGAs [42] inside NMSSMTools - for our benchmark point its numerical value seems smaller than the desired value $\Omega h^{2} \sim 0.12[43$, 44], which shows in any case that the relic density can be reduced sufficiently.

We have simulated events at the LHC at $8 \mathrm{TeV}$ for this point using MadGraph/MadEvent [45] which includes Pythia 6.4 [46] for showering and hadronisation. The emission of one additional hard jet was allowed in the simulation; the production cross sections for squark-squark, squark-gluino, squark-antisquark and gluino-gluino production were obtained by Prospino at NLO [47, 48], including correction factors from the resummation of soft gluon emmission estimated from $[49,50]$. (At $8 \mathrm{TeV}$, the total squark-gluino production cross section for the benchmark point is $\sim 524 \mathrm{fb}$.) The output in StdHEP format was given to CheckMATE [51] which includes the detector simulation DELPHES [52] and compares the signal rates to constraints in various search channels of ATLAS and CMS. Additional analyses were performed by means of MadAnalysis 5 [53, 54].

Following CheckMATE, the signal rates for the above benchmark point are compatible with constraints from available search channels for SUSY. In spite of the many $b$-jets from $H_{1}$ decays, the dominant constraints for this point originate from the search for jets and $E_{T}^{\text {miss }}$ in [1], more precisely from channel D requiring 5 hard jets, $E_{T}^{\text {miss }}>160 \mathrm{GeV}$ and $E_{T}^{\text {miss }} / m_{\text {eff }}(5 j)>0.2$, where $m_{\text {eff }}(N j)$ is the scalar sum of transverse momenta of the leading $\mathrm{N}$ jets and $E_{T}^{\mathrm{miss}}$.

Searches for events at the LHC with jets and $b \bar{b}$ pairs have also been performed by ATLAS in [55] however aiming at resonances in the $b \bar{b} b \bar{b}$ final state. Also the upper bounds on signal rates in $b \bar{b} \gamma \gamma$ final states from CMS [56] and ATLAS [57] are satisfied, amongst others since the $\gamma \gamma$ invariant mass required there does not cover our range of $M_{H_{1}}$.

An $M_{T 2}$ Higgs analysis was performed by CMS in [58], which aimed at a scenario similar to those discussed here: squark/gluino decay cascades ending in $\chi_{2}^{0} \rightarrow \chi_{1}^{0}+H_{S M}$. In one of the considered channels (high $H_{T}$ region) the cut on $E_{T}^{\text {miss }}$ was lowered to $E_{T}^{\text {miss }}>30 \mathrm{GeV}$. The absence of significant excesses was interpreted in terms of a gluino-induced simplified model leading to $M_{\text {gluino }} \gtrsim 850 \mathrm{GeV}$ (depending in $M_{\chi_{1}^{0}}$ ); however, $M_{\chi_{2}^{0}}=M_{\chi_{1}^{0}}+200 \mathrm{GeV}$ was assumed in this analysis.

Further searches for excesses in events with jets without lower cuts on $E_{T}^{\text {miss }}$ have been performed in [59-62]. The most constraining search channel in [59] is the one requiring 


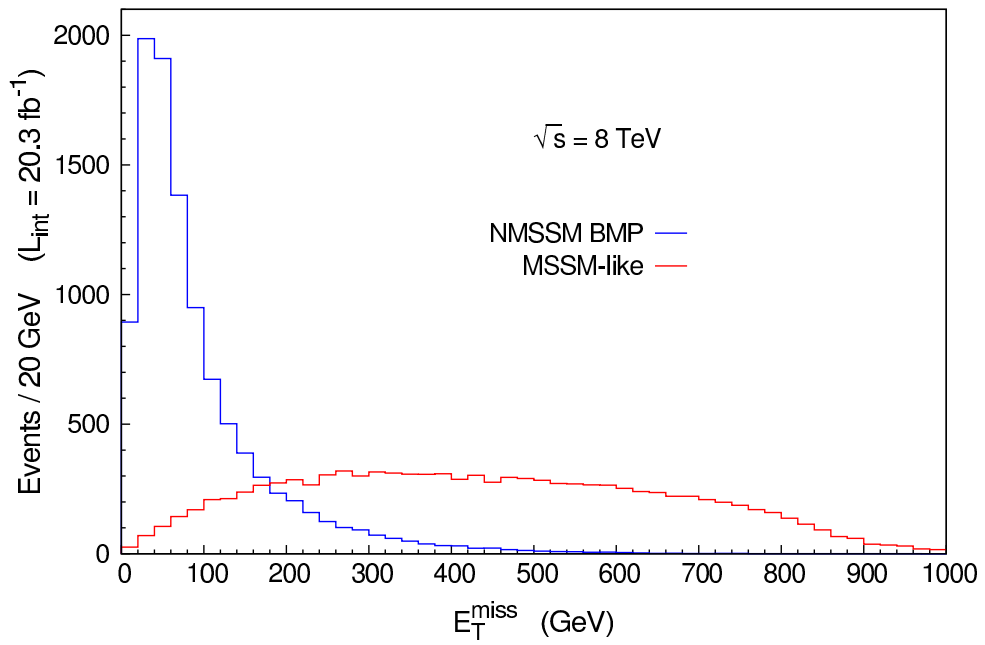

Figure 1. Spectra of $E_{T}^{\text {miss }}$ before cuts for the benchmark point (blue) and a similar point in the MSSM with a bino LSP (red).

7 jets with $p_{T}>80 \mathrm{GeV}$ and at least two tagged $b$-jets. After a simulation we find about 240 events in this channel for the benchmark point, complying with the data at the $2 \sigma$ level. Concerning the search for three-jet resonances in [60], we find that the two $b$-jets from $H_{1}$ decays merge sufficiently often into one single jet such that the event rates and acceptance are about 20 times smaller than the one assumed in [60] for gluino pair production with RPV decays into three jets, and the limits are well satisfied. Regarding the search for twojet resonances in [61] we find that the average two-jet mass peaks at $\sim 800 \mathrm{GeV}$ (somewhat below the squark/gluino masses) and the acceptance after cuts (within a window of a width of $\sim 15 \%$ times the mass) is $\lesssim 4 \%$; consequently the signal complies with the limits shown in figure 3 in [61]. Finally we have studied $S_{T}$, the sum of $\left|p_{T}\right|$ of objects with $\left|p_{T}\right|>50 \mathrm{GeV}$ relevant for the search for microscopic black holes in [62]. For all multiplicities $N \gtrsim 3 \ldots 10$ the signal events are below $10 \%$ of the data points shown in figures 2 and figures 3 in [62] without any peak-like structure, and thus this search is not restrictive. Hence present analyses, potentially sensitive to the decay products of two $H_{1}$ bosons in the final state, are not sensitive to the benchmark point.

Given that the masses of the gluino and the squarks of the first generation are well below $1 \mathrm{TeV}$ it is clear that a corresponding point in the parameter space of the MSSM would be well excluded, the reason being the different spectra of $E_{T}^{\text {miss }}$. To clarify this effect we show in figure 1 the spectrum of $E_{T}^{\text {miss }}$ for the benchmark point and for a similar point in the MSSM, which differs from the benchmark point only in a stable bino, which is now the LSP.

In figure 1 one sees the dramatic reduction of $E_{T}^{\text {miss }}$ due to the NLSP $\rightarrow$ LSP + $H_{1}$ decay; the few remaining events with large $E_{T}^{\text {miss }}$ for the benchmark point (denoted by NMSSM BMP in figure 1) originate from neutrinos from $\tau$ and $b$ decays after $H_{1} \rightarrow$ $\tau^{+} \tau^{-}, b \bar{b}$ (and, to a minor extent, from the singlino).

In fact, the final states from $H_{1}$ decays not only reduce $E_{T}^{\text {miss }}$, but lead also to an increase of $m_{\mathrm{eff}}(N j)$. Hence the cut $E_{T}^{\text {miss }} / m_{\mathrm{eff}}(5 j)>0.2$ in [1] reduces the number of 


\begin{tabular}{|c|c|c|c|c|c|c|c|c|c|}
\hline$M_{\tilde{S}}(\mathrm{GeV}):$ & 1 & 3 & 5 & 7 & 9 & 11 & 13 & 15 & 17 \\
\hline$M_{H_{1}}(\mathrm{GeV}):$ & \multicolumn{9}{|c|}{$R_{T}^{E_{T}^{\text {miss }}}:$} \\
\hline 87 & .125 & & & & & & & & \\
\hline 85 & .134 & .134 & & & & & & & \\
\hline 83 & .147 & .146 & .145 & & & & & & \\
\hline 81 & .166 & .169 & .161 & .160 & & & & & \\
\hline 79 & .192 & .194 & .186 & .186 & .179 & & & & \\
\hline 77 & .232 & .224 & .225 & .221 & .211 & .207 & & & \\
\hline 75 & .273 & .276 & .268 & .261 & .266 & .252 & .247 & & \\
\hline 73 & .319 & .316 & .309 & .310 & .307 & .302 & .298 & .294 & \\
\hline 71 & .358 & .366 & .362 & .359 & .353 & .355 & .353 & .345 & .343 \\
\hline
\end{tabular}

Table 2. Ratios $R_{T}^{E_{T}^{\text {miss }}}$ of the number of events with $E_{T}^{\text {miss }}>160 \mathrm{GeV}$ (before other cuts) in the NMSSM, over the number of events in the MSSM (with the bino as LSP), as function of $M_{\tilde{S}}$ and $M_{H_{1}}$ keeping $M_{\text {bino }}$ fixed at $89 \mathrm{GeV}$.

signal events even more dramatically by a factor $\sim 0.07$, and events passing this cut satisfy $E_{T}^{\text {miss }}>160 \mathrm{GeV}$ automatically. (For the MSSM-like point, channel D is actually not the most constraining channel, but rather channel AM.)

How sensitive are these reductions of signal events to the masses of the involved particles? In order to answer this question we have varied both the singlino mass $M_{\tilde{S}}$ from $1 \mathrm{GeV}$ to $17 \mathrm{GeV}$ and $M_{H_{1}}$ from $87 \mathrm{GeV}$ to $71 \mathrm{GeV}$, keeping the bino mass fixed at $89 \mathrm{GeV}$. We first studied the ratio $R_{T}^{E_{T}^{\text {miss }}}$ defined by the ratio of the number of events with $E_{T}^{\text {miss }}>160 \mathrm{GeV}$ (before other cuts) in the NMSSM, over the number of events in the MSSM with the bino as LSP. The results for $R_{T}^{E_{T}^{\text {miss }}}$ are shown in table 2. (The relative statistical error on $R_{T}^{E_{T}^{\text {miss }}}$ is about $2 \%$ for $R^{E_{T}^{\text {miss }}} \sim 0.15$, decreasing slightly with increasing $R_{T}^{E_{T}^{\text {miss }}}$.)

We see that, for the singlino mass $M_{\tilde{S}}$ in the kinematically allowed range, $R^{E_{T}^{\text {miss }}}$ varies little with $M_{\tilde{S}}$ for fixed $M_{H_{1}}$ : on average, $R_{T}^{E_{T}^{\text {miss }}}$ decreases slightly with increasing $M_{\tilde{S}}$ towards the boundary of phase space. On the other hand, for fixed $M_{\tilde{S}}, R^{E_{T}^{\text {miss }}}$ has a stronger increase with decreasing $M_{H_{1}}$ (away from the boundary of phase space).

As stated above, the impact of the "missing" $E_{T}^{\text {miss }}$ on the signal rates in channel D [1] after all cuts including $E_{T}^{\text {miss }} / m_{\text {eff }}(5 j)>0.2$ is actually stronger. In figure 2 we show the ratio of signal events in the NMSSM over the number of events in the MSSM with the bino as LSP, after all cuts for this channel, as function of $M_{H_{1}}$ (keeping the singlino mass fixed at the value of the benchmark point of $5.3 \mathrm{GeV}$ ). The error bars indicate the statistical fluctuations from our simulations as determined by CheckMATE.

We see that the reduction of signal events remains very strong, even if $M_{H_{1}}$ is several $\mathrm{GeV}$ away from the boundary of phase space. Hence the result of the analyses summarised in table 2 and figure 2 is that suppressing the number of signal events in typical SUSY search channels does not require a particular fine-tuning of masses.

On the other hand we should keep in mind that contributions from neutrinos from squark decay cascades to $E_{T}^{\text {miss }}$ are suppressed (due to the heavy charginos/extra neutrali- 


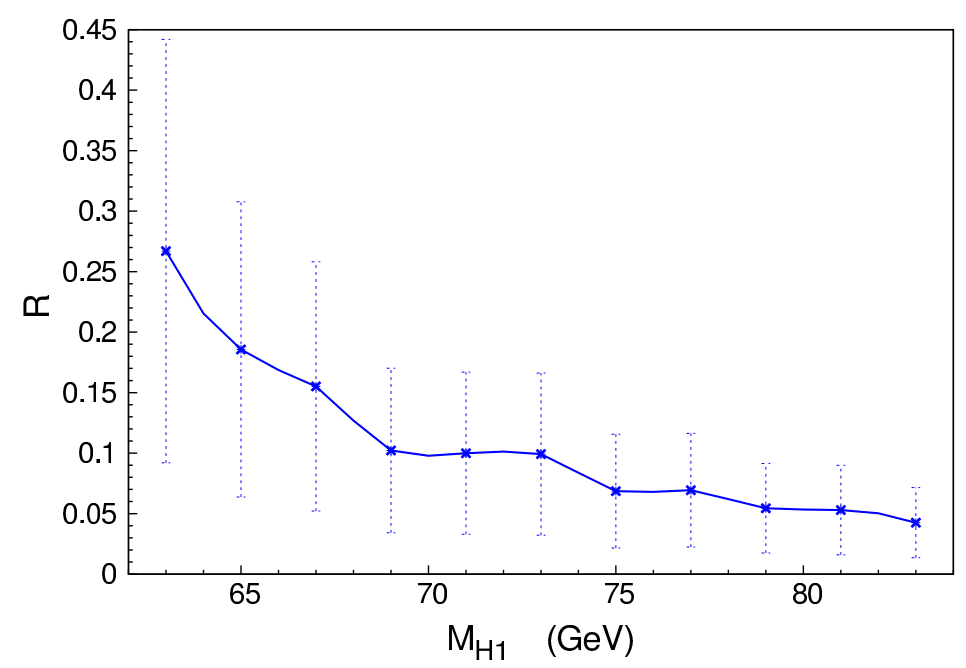

Figure 2. The ratio $R$ of signal events in the NMSSM over the number of events in the MSSM with the bino as LSP, after all cuts for channel D in [1], as function of $M_{H_{1}}$ keeping the singlino mass fixed at $5.3 \mathrm{GeV}$.

nos) for all points considered above. Otherwise, as we have checked, searches for jets and $E_{T}^{\text {miss }}$ (typically from [1]) exclude squarks/gluinos with masses below $1 \mathrm{TeV}$ due to contributions from neutrinos from squark decay cascades to $E_{T}^{\text {miss }}$, even if the LSPs contribute little to $E_{T}^{\text {miss }}$.

The next question is whether other search strategies, not relying on strong cuts on $E_{T}^{\text {miss }}$, can be sensitive to such difficult SUSY scenarios with two $H_{1}$ bosons in the final state. A first proposal towards extracting corresponding signals - which will be improved in a future publication - is presented in the next section.

\section{Towards the extraction of signals in $b \bar{b}+\tau^{+} \tau^{-}$final states at $14 \mathrm{TeV}$}

Signals from Higgs production via neutralino decays in sparticle decay cascades have been analysed previously, mostly in the context of the MSSM in [17, 63-72]. There, however, significant lower cuts on $E_{T}^{\text {miss }}$ were applied, since in the considered scenarios the LSP had no reason to be particularly soft.

In the present scenario the final states of squark and gluino production are characterised by jets with large $p_{T}$, little $E_{T}^{\text {miss }}$, but remnants of two $H_{1}$ Higgs bosons. The couplings of $H_{1}$ to SM particles originate from a small mixing of $H_{1}$ with the SM-like Higgs boson (since the third CP-even Higgs state is very heavy). Hence its branching ratios coincide - up to kinematic suppressions of decays into $W W^{*} / Z Z^{*}$ — with those of the SM-like Higgs boson; we obtain $B R\left(H_{1} \rightarrow b \bar{b}\right) \sim 0.85$. The coupling of $H_{1}$ to two light singlet-like CP-odd states $A_{1}$ is proportional to the squares of the NMSSM-specific Yukawa couplings $\lambda, \kappa$ which are small (see table 1); accordingly the branching fraction $B R\left(H_{1} \rightarrow A_{1} A_{1}\right) \sim 5 \times 10^{-6}$ is neglibly small. (Due to the smallness of the mixing the prospects for a direct discovery of $H_{1}$ at the LHC are rather dim: its couplings to SM particles squared, and hence its 


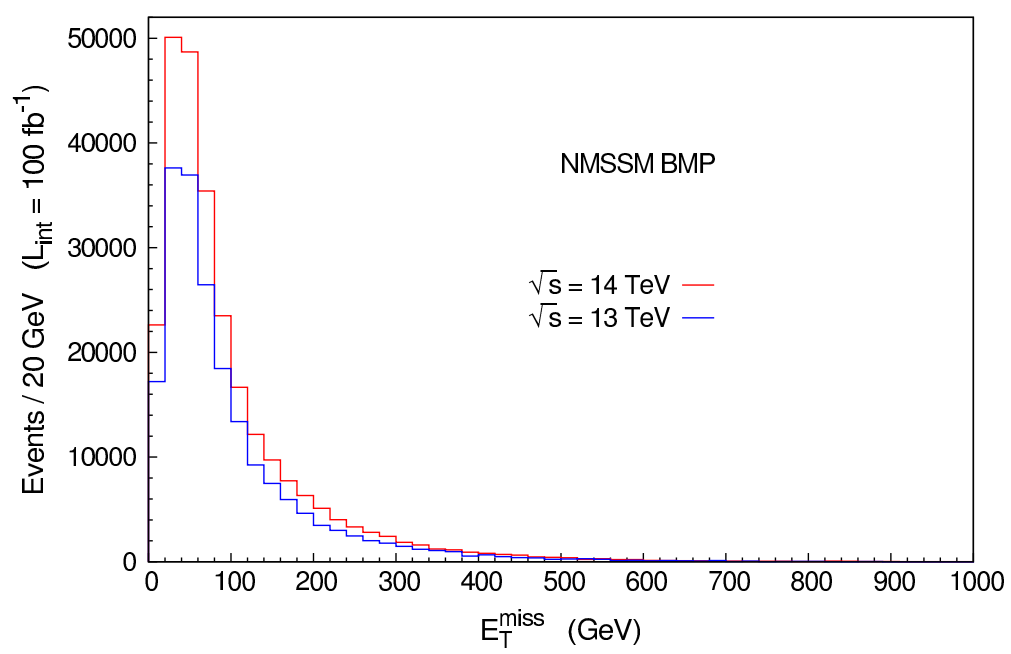

Figure 3. Expected $E_{T}^{\text {miss }}$ distribution for the benchmark point at the LHC at 13 and $14 \mathrm{TeV}$ c.m. energy.

production cross sections and signal rates, are only $6 \%-6.5 \%$ of the ones of a SM-like Higgs boson of similar mass.)

Subsequently we describe an approach towards the extraction of a possible signal in the $b \bar{b}+\tau^{+} \tau^{-}$final state, using the same simulation methods described in the previous section.

First we have studied $E_{T}^{\text {miss }}$ for the benchmark point for $p p$ collisions at the LHC at $13 \mathrm{TeV}$ and $14 \mathrm{TeV}$ c.m. energy assuming an integrated luminosity of $100 \mathrm{fb}^{-1}$. As can be seen in figure $3, E_{T}^{\text {miss }}$ is still peaked at low values.

When looking for the remnants of two $H_{1}$ Higgs bosons, an important question is which transverse momenta can one expect for these particles. In figure 4 we show the leading and next-to-leading $p_{T}$ distribution corresponding to the benchmark point. (Here and in the following we concentrate on $14 \mathrm{TeV}$ c.m. energy.) We see that the $p_{T}$ of the leading $H_{1}$ is peaked near $400 \mathrm{GeV}$, and the $p_{T}$ of the next-to-leading $H_{1}$ is peaked near $200 \mathrm{GeV}$, which can be used for cuts on the final states.

We next describe the sequence of cuts which were applied. The analysis of the events was performed in two steps: to start with, jets were constructed by Fastjet [73] (part of the Delphes package [52]) using the anti- $k_{T}$ algorithm [74] and a jet cone radius $R=0.5$. This value was chosen such that, as often as possible, all decay products of the leading $H_{1}$ (but no other hadrons) are part of a single jet whose mass distribution is analysed at the end.

We require four hard jets (including $b$-tagged jets) with $p_{T}>$ $400 \mathrm{GeV}, 200 \mathrm{GeV}, 80 \mathrm{GeV}, 80 \mathrm{GeV}$, respectively. A significant $E_{T}^{\text {miss }}$ is not part of the signal; some $E_{T}^{\text {miss }}$ can be expected, however, from neutrinos of $\tau$ decays once we require $2 \tau$ in the final state (see figure 3 ). Hence we only impose $E_{T}^{\text {miss }}>30 \mathrm{GeV}$.

Then, jets of the same event were reconstructed with a jet cone radius $R=0.15$. The aim is to identify as many "slim" $b$-jets as possible, together with their kinematic properties. The value $R=0.15$ is just marginally larger than the granularity $0.1 \times 0.1$ of 


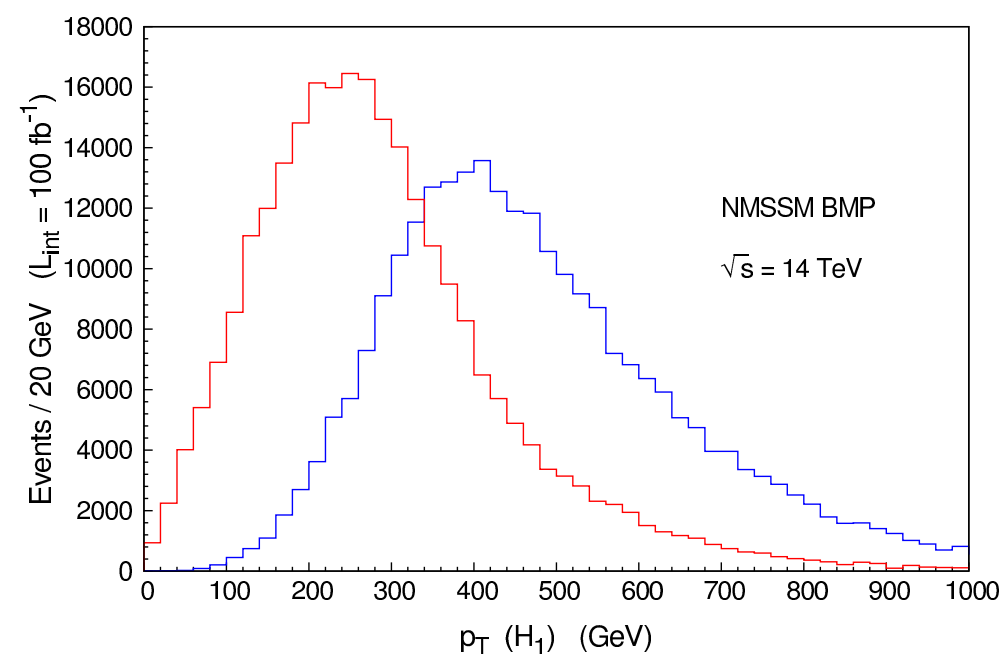

Figure 4. Transverse momentum distributions of the leading $H_{1}$ (blue) and next-to-leading $H_{1}$ (red) after squark and gluino production at the LHC at $14 \mathrm{TeV}$ c.m. energy for the benchmark point.

the ATLAS hadronic tile calorimeter (we use the ATLAS detector card inside Delphes). For $b$-tagged jets we require $p_{T}>40 \mathrm{GeV}$ and assume a $b$-tag efficiency of $70 \%$ (mistag efficiencies from $c$-jets of $10 \%$, and from light quark/gluon jets of $1 \%$ ).

Among the jets reconstructed with $R=0.15$ we require $\geq 2 b$-jets and $\geq 2$ hadronic $\tau$ leptons. Since the invariant mass of the pair of $\tau$-leptons is difficult to reconstruct we just require $M_{\tau \tau}<120 \mathrm{GeV}$ and, in order to remove the background from fake $\tau$ leptons (see below), $M_{\tau \tau}>20 \mathrm{GeV}$.

The $2 b$-jets next to each other (with the smallest $\Delta R$ ) are combined into a $2 b$ pseudojet, 2bPJ.

Among the jets constructed with a jet cone radius $R=0.5$ we look for the one closest to the $2 \mathrm{bPJ}$ (as close as $\Delta R<0.1$ ); this jet $\widehat{J}$ is our candidate for the remnants of $H_{1} \rightarrow b \bar{b}$. For $\widehat{J}$ we further require $p_{T}>400 \mathrm{GeV}$. Since $\widehat{J}$ is assumed to include the $2 \mathrm{bPJ}$, the invariant mass of the $2 \mathrm{bPJ}$ should be smaller than the mass of $\widehat{J}$. (The mass of the $2 \mathrm{bPJ}$ can be considerably smaller due to radiation off the $b$-quarks not included in the $R=0.15$ jets.) Finally we require the mass of $\widehat{J}$ to be $40 \mathrm{GeV}<M_{\widehat{J}}<120 \mathrm{GeV}$, and plot $M_{\widehat{J}}$ in this range.

The result displayed in figure 5, which is based on the simulation of $\sim 230000$ events, shows that $M_{\widehat{J}}$ peaks indeed near the mass of $H_{1}, 83 \mathrm{GeV}$ for the benchmark point considered here. The event rates are normalised to an integrated luminosity of $100 \mathrm{fb}^{-1}$; for the total squark-gluino production cross section at $14 \mathrm{TeV}$ we have $5232 \mathrm{fb}$ at NLO+NNLO. The impact of the above cuts is shown in table 3 ; within the signal region $40 \mathrm{GeV}<M_{\widehat{J}}<120 \mathrm{GeV}$ the cross section is about $23 \mathrm{fb}$. Given the $H_{1} \rightarrow \tau^{+} \tau^{-}$branching fraction of about $8 \%$ and the tagging efficiencies, the dominant reduction of signal events results from the requested $\geq 2 b$-jets and $\geq 2 \tau$ leptons.

A priori, the following Standard Model backgrounds contribute to the final states defined above: $j j b \bar{b} \tau^{+} \tau^{-}$from QCD and electroweak production, $j j t \bar{t}$ possibly with addi- 


\begin{tabular}{|c|c|c|}
\hline & Benchmark point & $j j b \bar{b}$ background \\
\hline Cross section in fb & 5232 & $1.47 \times 10^{5}$ \\
\hline$p_{T}($ jets $)>400,200,80,80 \mathrm{GeV}$ & 3513 & 6835 \\
\hline$E_{T}^{\text {miss }}>30 \mathrm{GeV}$ & 3118 & 3875 \\
\hline$\geq 2 b$-jets, $\geq 2 \tau_{h}$ with $20 \mathrm{GeV}<M_{\tau \tau}<120 \mathrm{GeV}$ & 99.3 & 97.7 \\
\hline$\Delta R(\widehat{J}, 2 \mathrm{bPJ})<0.1$ & 48.9 & 37.8 \\
\hline$p_{T}(\widehat{J})>400 \mathrm{GeV}$ & 27.1 & 12.9 \\
\hline$M_{2 \mathrm{bPJ}}<M_{\widehat{J}}$ & 24.1 & 9.6 \\
\hline $40 \mathrm{GeV}<M_{\widehat{J}}<120 \mathrm{GeV}$ & 22.8 & 7.5 \\
\hline
\end{tabular}

Table 3. Impact of the cuts described in the text on the event rates of the benchmark point and the dominant $j j b \bar{b}$ background (the latter after cuts at the parton level as described in the text).

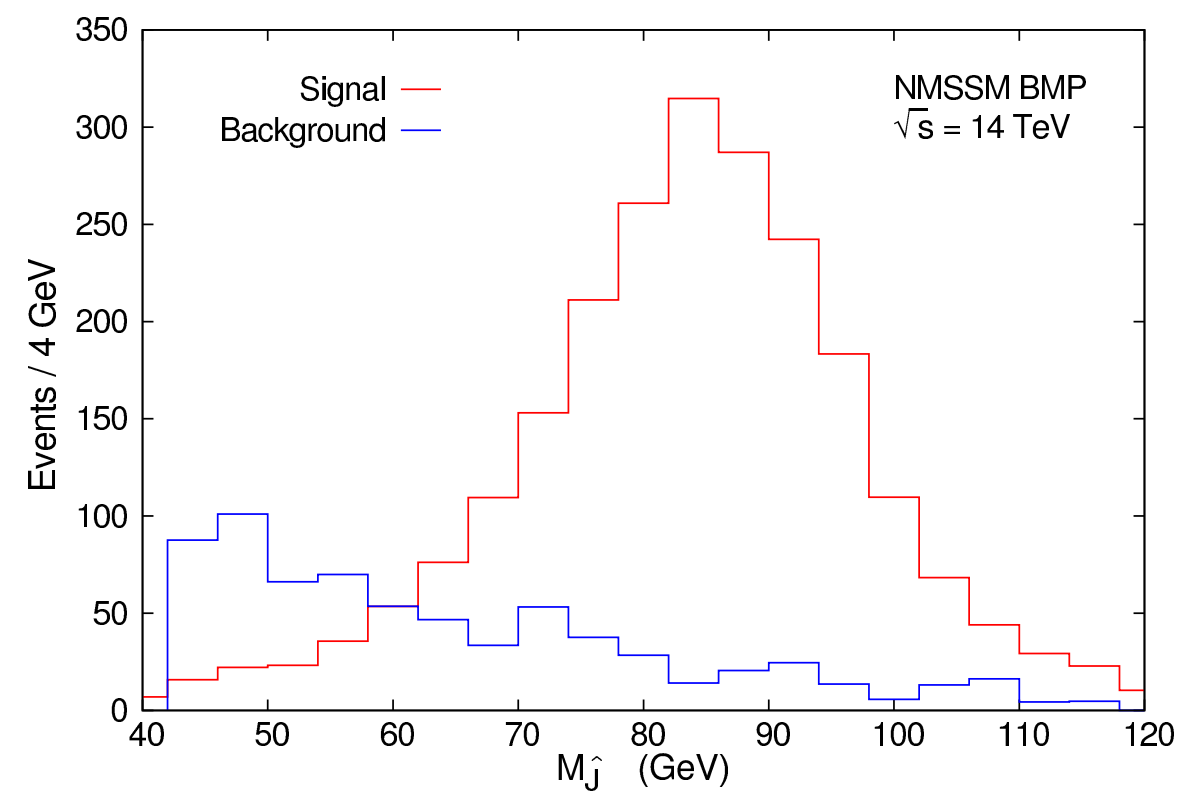

Figure 5. Plot of $M_{\widehat{J}}$ (in red) for the benchmark point at $14 \mathrm{TeV}$ c.m. energy, after application of the cuts described in the text and in table 3 . The background contribution from $j j b \bar{b}$ with two mistagged $\tau$ leptons is shown in blue.

tional mistagged $\tau$ leptons, and $j j b \bar{b}$ with two mistagged $\tau$ leptons. We have estimated these backgrounds using the same simulation methods applied for the signal. Since we require four well separated hard jets in the final state, each jet is assumed to originate from a corresponding parton (quark or gluon for the jets " $j$ "). In order to make our cut analysis sufficiently efficient, we also applied cuts at the parton level in MadGraph on jets and $b$-quarks: for $b$-quarks we required $p_{T}>100 \mathrm{GeV}$ and $|\eta|<2$, and for the four leading jets (including $b$-quarks) $p_{T}>200 \mathrm{GeV}, 100 \mathrm{GeV}, 80 \mathrm{GeV}, 80 \mathrm{GeV}$, respectively. We checked that these cuts do not generate a bias in the $M_{\widehat{J}}$ spectrum after applying the additional cuts described above and in table 3 .

After the cuts, the background from $j j b \bar{b} \tau^{+} \tau^{-}$from QCD and electroweak production turned out to be negligibly small, with a cross section in the signal region of about $0.007 \mathrm{fb}$. 


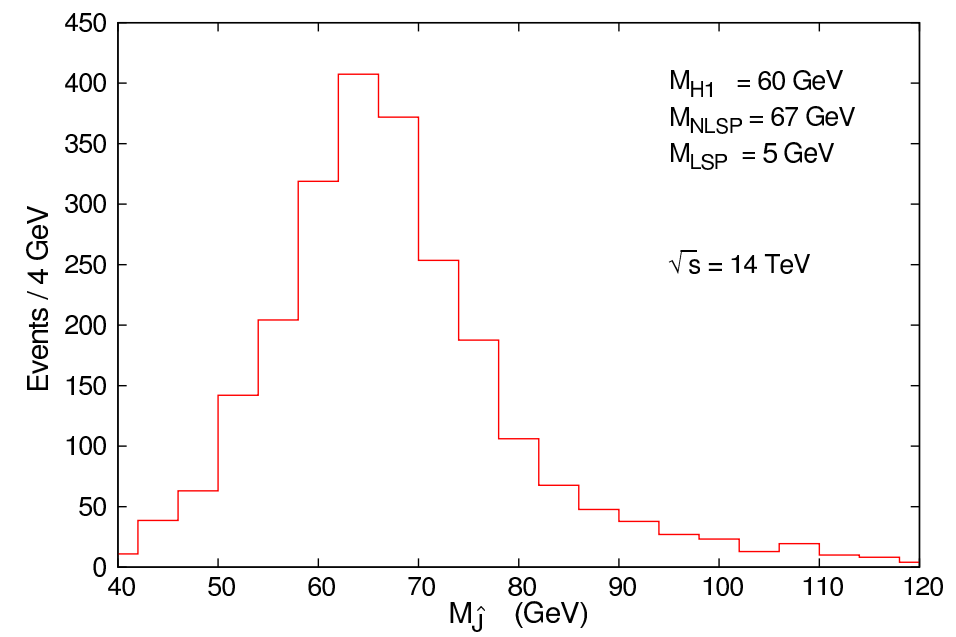

Figure 6. Plot of $M_{\widehat{J}}$ at $14 \mathrm{TeV}$ c.m. energy, for a point with $M_{H_{1}}=60 \mathrm{GeV}, M_{\mathrm{NLSP}}=67 \mathrm{GeV}$ and $M_{\mathrm{LSP}}=5 \mathrm{GeV}$ after application of the cuts described in the text and in table 3 .

After all cuts, the background from $j j t \bar{t}$ is also small, with a cross section in the signal region of about $0.44 \mathrm{fb}$.

However, the background from $j j b \bar{b}$ with two mistagged $\tau$ leptons is relatively large due to the fact that we tag for $b$-jets and $\tau$-leptons using jet reconstruction with a small jet cone radius $R=0.15$. Many of such "slim" jets are mistagged as $\tau$-leptons, often as pairs with a relatively low invariant mass. A priori, about $5 \%$ of all $j j b \bar{b}$ events after cuts contain such a fake $\tau$ pair. This fake rate can be reduced by a factor $\sim \frac{1}{2}$ after a cut $M_{\tau \tau}>20 \mathrm{GeV}$, which reduces the signal by only about $12 \%$. Then this background results in a cross section in the signal region of about $7 \mathrm{fb}$. Its contribution to $M_{\widehat{J}}$, based on the simulation of 300000 events, is also shown in figure 5, and it seems that the signal can be distinguished clearly from this background.

In practise the background is often obtained from data-driven control regions. Once it is measured, modifications of the cuts given above and/or additional cuts (for example on the absence of isolated leptons) are likely to improve the signal/background ratio even further.

Of course, the mass $M_{H_{1}}$ can differ from the value $M_{H_{1}}=83 \mathrm{GeV}$ assumed for the benchmark point. In order to see the impact of a lighter $H_{1}$ we have repeated the simulation for a point with the same sparticle spectrum but $M_{H_{1}}=60 \mathrm{GeV}, M_{\mathrm{NLSP}}=67 \mathrm{GeV}$ and $M_{\mathrm{LSP}}=5 \mathrm{GeV}$. The resulting $M_{\widehat{J}}$ spectrum is shown in figure 6 and again we see that the sequence of cuts allows, in principle, to identify $M_{H_{1}}$ from the $M_{\widehat{J}}$ spectrum.

The $H_{1} H_{1}$ final state, characteristic of squark/gluino production in the present scenario, resembles actually Higgs pair production in the Standard Model up to the unknown $H_{1}$ mass but, provided that squarks and gluinos are not excessively heavy, with an associated larger cross section (see [75-79] for some recent studies in the Standard Model). Corresponding techniques like more refined subjet-based analyses applied to the $b \bar{b} \tau^{+} \tau^{-}$ final state in [75] can probably be useful here as well.

The squark/gluino production cross sections would decrease, of course, for squarks and/or gluinos heavier than their benchmark value $(860 / 890 \mathrm{GeV}$, respectively), and the 


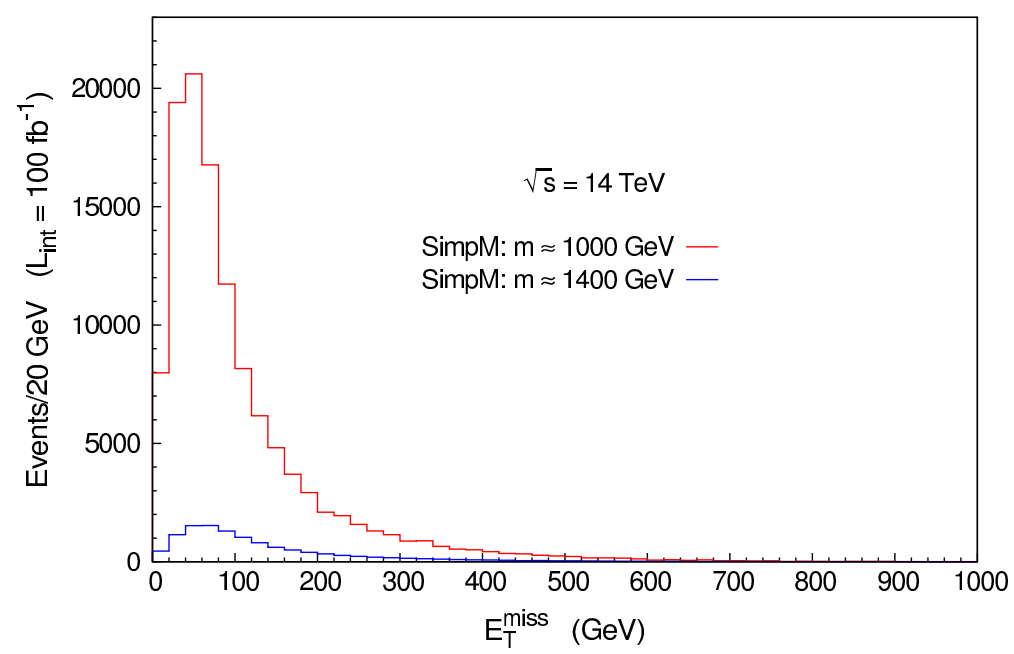

Figure 7. Expected $E_{T}^{\text {miss }}$ distribution at the LHC at $14 \mathrm{TeV}$ c.m. energy for two simplified models with squark $\sim$ gluino masses of $1000 \mathrm{GeV}$ and $1400 \mathrm{GeV}$.

kinematics will change. First we investigate the impact of heavier squarks and gluinos on the shape of $E_{T}^{\text {miss }}$. We illustrate this with simplified models where, as in the case of the previous benchmark point, squarks decay with a $100 \%$ branching ratio into the bino-like NLSP (still with a mass of $89 \mathrm{GeV}$ ) which can only decay (with $100 \% \mathrm{BR}$ ) into $H_{1}$ and the singlino-like LSP (both still with masses of $83 \mathrm{GeV}$ and $5 \mathrm{GeV}$, respectively). The corresponding $E_{T}^{\text {miss }}$ distributions are shown in figure 7 for squark/gluino masses of $1000 \mathrm{GeV}$ or $1400 \mathrm{GeV}$. (The gluinos are taken $5 \mathrm{GeV}$ heavier than squarks to allow, for simplicity, for flavour democratic gluino 3-body decays into quarks + squarks of the first two generations.)

One finds that $E_{T}^{\text {miss }}$ still strongly peakes at low values; hard cuts on $E_{T}^{\text {miss }}$ would remove again most of the signal events. The transverse momenta of the the leading and next-to-leading $H_{1}$ for squarks/gluinos with masses of $1000 \mathrm{GeV}$ and $1400 \mathrm{GeV}$, respectively, are shown in figure 8. As visible from figure 8 , the average transverse momenta of the $H_{1}$ states are considerably larger for heavier squarks/gluinos.

Finally we ask whether the shape of the $M_{\widehat{J}}$ spectrum changes for heavier squarks/gluinos. Using the same analysis and cuts as before, the resulting $M_{\widehat{J}}$ spectrum is shown in figure 9 for squark/gluino masses of $1000 \mathrm{GeV}, 1200 \mathrm{GeV}$ and $1400 \mathrm{GeV}$. We see that the shape of the $M_{\widehat{J}}$ spectrum remains unchanged; only the signal rate decreases as expected (slightly less, in fact) as does the production cross section which is now about $2226 \mathrm{fb}, 693 \mathrm{fb}$ and $242 \mathrm{fb}$ for squark/gluino masses of $1000 \mathrm{GeV}, 1200 \mathrm{GeV}$ and $1400 \mathrm{GeV}$, respectively. However, for squark/gluino masses above $1400 \mathrm{GeV}$, the signal obtained with the present cuts (and jet analysis) starts to fall below the background from $j j b \bar{b}$ with two mistagged $\tau$ leptons (shown in figure 5).

On the other hand, since the production of heavier squarks and gluinos generates both $H_{1}$ states and jets with larger transverse momenta, cuts can be optimised. Search channels with significantly harder cuts on the transverse momenta of at least the candidate $\widehat{J}$-jets 


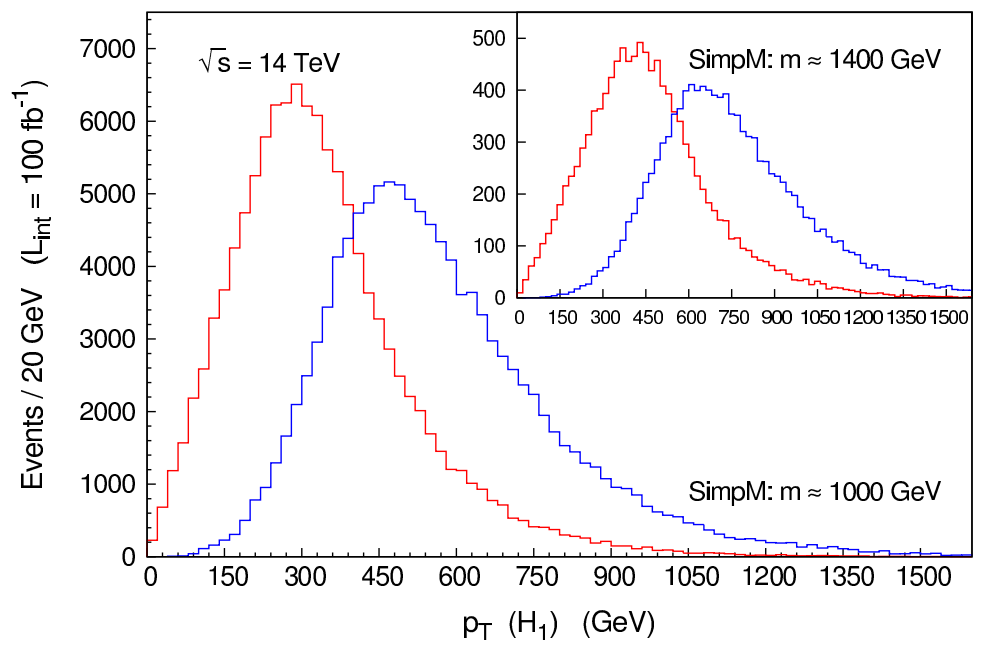

Figure 8. Transverse momentum distributions of the leading $H_{1}$ (blue) and next-to-leading $H_{1}$ (red) after squark and gluino production at the LHC at $14 \mathrm{TeV}$ c.m. energy. We assume simplified spectra, with squarks/gluino masses of $1000 \mathrm{GeV}$ and $1400 \mathrm{GeV}$.

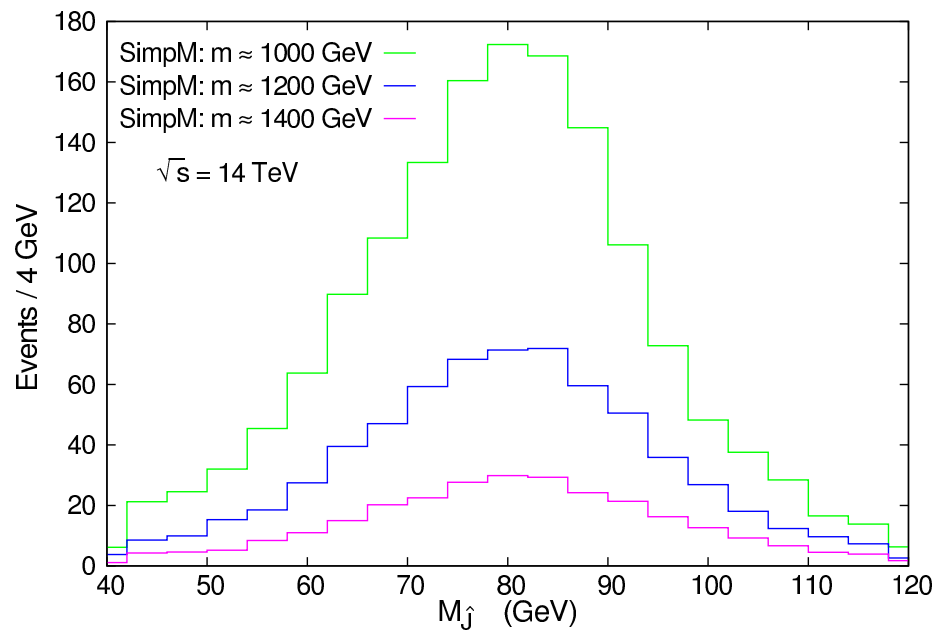

Figure 9. $M_{\widehat{J}}$ at $14 \mathrm{TeV}$ c.m. energy for simplified model spectra with $M_{H_{1}}=83 \mathrm{GeV}, M_{\mathrm{NLSP}}=$ $89 \mathrm{GeV}$ and $M_{\mathrm{LSP}}=5 \mathrm{GeV}$, and squark/gluino masses of $1000 \mathrm{GeV}, 1200 \mathrm{GeV}$ and $1400 \mathrm{GeV}$, respectively.

(assumed to contain the remnants of the leading $H_{1}$ ) can be employed. Corresponding analyses will be the subject of future publications.

\section{Conclusions and outlook}

The most important result of the present paper is the existence of scenarios in the general NMSSM in which a light singlino at the end of sparticle decay cascades reduces strongly the missing transverse energy, one of the essential criteria in standard search channels for supersymmetry. In such "worst case scenarios" hardly any missing transverse energy is produced along each step of sparticle decay cascades. We present a realistic benchmark 
point, consistent with the properties of the Standard Model-like Higgs boson at $\sim 126 \mathrm{GeV}$ and the dark matter relic density, satisfying present constraints from SUSY search channels with all sparticle masses below $\sim 1 \mathrm{TeV}$.

The two NMSSM-like Higgs bosons $H_{1}$, produced in each event of squark, squarkgluino or gluino pair production in this scenario, allow for new search channels which do not rely on large missing transverse energy, but on the $H_{1}$ decay products. We have presented an analysis which shows that, for not too heavy squarks/gluinos, a $H_{1}$ signal can be visible above the Standard Model background in the $b \bar{b} \tau^{+} \tau^{-}+$jets final state. This analysis can certainly be improved in various aspects, but already indicates the lines along which a signal can be obtained.

Among the possible improvements are analyses based on jet substructure as is the case of Higgs pair production into the same final state in [75] (replacing the step of our analysis based on a jet cone radius of 0.15 ), which may also help to reduce the background from mistagged tau pairs. Also searches for a $(b \bar{b})(b \bar{b})$ final state as in [79] might be feasible. Finally, in order to get some direct information on the masses of the originally produced squark/gluino pairs (beyond the production cross section), analyses based on jet substructure may be combined with analyses based on $M_{T 2}$ as, for example, in [58].

Variants of the benchmark scenarios discussed here could also be realised in principle: first, winos and/or higgsinos could be lighter and appear in squark decay cascades. Then standard SUSY search channels relying on $E_{T}^{\text {miss }}$ and jets, isolated leptons etc., start to become sensitive to squark/gluino production but it remains to be studied when, in the presence of a light singlino and for the kinematical situation discussed here, such search channels become more sensitive than the type of analysis presented here.

Second, the final state " $X$ " in the final step NLSP $\rightarrow X+$ LSP of sparticle decay cascades does not necessarily have to be a light NMSSM-specific Higgs boson $H_{1}$. Again, if $X$ is for instance a $Z$ boson or a combination of $Z$ and $H_{\mathrm{SM}}$ bosons (depending on the branching fractions of the NLSP), standard SUSY search channels can become relevant since more $E_{T}^{\text {miss }}$ is expected from $X$ decays. However, if the number of events with $E_{T}^{\text {miss }}$ is still reduced due the particular kinematical situation discussed here, channels which depend less on $E_{T}^{\text {miss }}$ but more on $X$ decay products would again be more promising. Such cases merit also to be studied in the future.

\section{Acknowledgments}

We are grateful to Y. Kats for helpful comments on additional searches for new physics. We acknowledge help from A. Belyaev for background simulations, and thank B. Fuks, S. Kulkarni and S. Moretti for helpful discussions. A. M. T. and U. E. acknowledge support from European Union Initial Training Network INVISIBLES (PITN-GA-2011289442). U. E. acknowledges support from the ERC advanced grant Higgs@LHC, and from the European Union Initial Training Network HiggsTools (PITN-GA-2012-316704).

Open Access. This article is distributed under the terms of the Creative Commons Attribution License (CC-BY 4.0), which permits any use, distribution and reproduction in any medium, provided the original author(s) and source are credited. 


\section{References}

[1] ATLAS collaboration, Search for squarks and gluinos with the ATLAS detector in final states with jets and missing transverse momentum and $20.3 \mathrm{fb}^{-1}$ of $\sqrt{\mathrm{s}}=8$ TeV proton-proton collision data, ATLAS-CONF-2013-047.

[2] CMS collaboration, Search for new physics in the multijet and missing transverse momentum final state in proton-proton collisions at $\sqrt{s}=8 \mathrm{TeV}$, JHEP 06 (2014) 055 [arXiv: 1402.4770] [INSPIRE].

[3] I. Melzer-Pellmann and P. Pralavorio, Lessons for SUSY from the LHC after the first run, Eur. Phys. J. C 74 (2014) 2801 [arXiv:1404.7191] [INSPIRE].

[4] J.L. Feng, Naturalness and the Status of Supersymmetry, Ann. Rev. Nucl. Part. Sci. 63 (2013) 351 [arXiv: 1302.6587] [INSPIRE].

[5] U. Ellwanger, C. Hugonie and A.M. Teixeira, The Next-to-Minimal Supersymmetric Standard Model, Phys. Rept. 496 (2010) 1 [arXiv:0910.1785] [INSPIRE].

[6] L.J. Hall, D. Pinner and J.T. Ruderman, A Natural SUSY Higgs Near 126 GeV, JHEP 04 (2012) 131 [arXiv:1112.2703] [InSPIRE].

[7] U. Ellwanger, A Higgs boson near 125 GeV with enhanced di-photon signal in the NMSSM, JHEP 03 (2012) 044 [arXiv: 1112.3548] [INSPIRE].

[8] A. Arvanitaki and G. Villadoro, A Non Standard Model Higgs at the LHC as a Sign of Naturalness, JHEP 02 (2012) 144 [arXiv:1112.4835] [INSPIRE].

[9] S.F. King, M. Muhlleitner and R. Nevzorov, NMSSM Higgs Benchmarks Near 125 GeV, Nucl. Phys. B 860 (2012) 207 [arXiv:1201.2671] [InSPIRE].

[10] Z. Kang, J. Li and T. Li, On Naturalness of the MSSM and NMSSM, JHEP 11 (2012) 024 [arXiv:1201.5305] [INSPIRE].

[11] J.-J. Cao, Z.-X. Heng, J.M. Yang, Y.-M. Zhang and J.-Y. Zhu, A SM-like Higgs near 125 GeV in low energy SUSY: a comparative study for MSSM and NMSSM, JHEP 03 (2012) 086 [arXiv: 1202.5821] [INSPIRE].

[12] U. Ellwanger and C. Hugonie, Neutralino cascades in the $(M+1) S S M$, Eur. Phys. J. C 5 (1998) 723 [hep-ph/9712300] [INSPIRE].

[13] U. Ellwanger and C. Hugonie, Topologies of the $(M+1)$ SSM with a singlino LSP at LEP-2, Eur. Phys. J. C 13 (2000) 681 [hep-ph/9812427] [INSPIRE].

[14] A. Dedes, C. Hugonie, S. Moretti and K. Tamvakis, Phenomenology of a new minimal supersymmetric extension of the standard model, Phys. Rev. D 63 (2001) 055009 [hep-ph/0009125] [INSPIRE].

[15] S.Y. Choi, D.J. Miller and P.M. Zerwas, The Neutralino sector of the next-to-minimal supersymmetric standard model, Nucl. Phys. B 711 (2005) 83 [hep-ph/0407209] [InSPIRE].

[16] K. Cheung and T.-J. Hou, Light Pseudoscalar Higgs boson in Neutralino Decays in the Next-to-Minimal Supersymmetric Standard Model, Phys. Lett. B 674 (2009) 54 [arXiv:0809.1122] [INSPIRE].

[17] O. Stal and G. Weiglein, Light NMSSM Higgs bosons in SUSY cascade decays at the LHC, JHEP 01 (2012) 071 [arXiv:1108.0595] [INSPIRE]. 
[18] D.G. Cerdeño, P. Ghosh, C.B. Park and M. Peiró, Collider signatures of a light NMSSM pseudoscalar in neutralino decays in the light of LHC results, JHEP 02 (2014) 048 [arXiv: 1307.7601] [INSPIRE].

[19] D. Das, U. Ellwanger and A.M. Teixeira, Modified Signals for Supersymmetry in the NMSSM with a Singlino-like LSP, JHEP 04 (2012) 067 [arXiv:1202.5244] [INSPIRE].

[20] D. Das, U. Ellwanger and A.M. Teixeira, LHC constraints on $M_{1 / 2}$ and $m_{0}$ in the semi-constrained NMSSM, JHEP 04 (2013) 117 [arXiv:1301.7584] [INSPIRE].

[21] H. Baer, H. Prosper and H. Summy, Early SUSY discovery at CERN LHC without missing E(T): The Role of multi-leptons, Phys. Rev. D 77 (2008) 055017 [arXiv:0801.3799] [INSPIRE].

[22] H. Baer, V. Barger, A. Lessa and X. Tata, Supersymmetry discovery potential of the LHC at $\sqrt{s}=10 \mathrm{TeV}$ and 14-TeV without and with missing E(T), JHEP 09 (2009) 063 [arXiv:0907.1922] [INSPIRE].

[23] M. Lisanti, P. Schuster, M. Strassler and N. Toro, Study of LHC Searches for a Lepton and Many Jets, JHEP 11 (2012) 081 [arXiv:1107.5055] [INSPIRE].

[24] J. Fan, M. Reece and J.T. Ruderman, Stealth Supersymmetry, JHEP 11 (2011) 012 [arXiv: 1105.5135] [INSPIRE].

[25] J. Fan, M. Reece and J.T. Ruderman, A Stealth Supersymmetry Sampler, JHEP 07 (2012) 196 [arXiv: 1201.4875] [INSPIRE].

[26] J.A. Evans, Y. Kats, D. Shih and M.J. Strassler, Toward Full LHC Coverage of Natural Supersymmetry, JHEP 07 (2014) 101 [arXiv:1310.5758] [INSPIRE].

[27] S.P. Martin, Compressed supersymmetry and natural neutralino dark matter from top squark-mediated annihilation to top quarks, Phys. Rev. D 75 (2007) 115005 [hep-ph/0703097] [INSPIRE].

[28] T.J. LeCompte and S.P. Martin, Large Hadron Collider reach for supersymmetric models with compressed mass spectra, Phys. Rev. D 84 (2011) 015004 [arXiv:1105.4304] [INSPIRE].

[29] T.J. LeCompte and S.P. Martin, Compressed supersymmetry after 1/fb at the Large Hadron Collider, Phys. Rev. D 85 (2012) 035023 [arXiv:1111.6897] [InSPIRE].

[30] G. Bélanger, M. Heikinheimo and V. Sanz, Model-Independent Bounds on Squarks from Monophoton Searches, JHEP 08 (2012) 151 [arXiv: 1205.1463] [INSPIRE].

[31] H.K. Dreiner, M. Krämer and J. Tattersall, How low can SUSY go? Matching, monojets and compressed spectra, Europhys. Lett. 99 (2012) 61001 [arXiv:1207.1613] [INSPIRE].

[32] B. Bhattacherjee, A. Choudhury, K. Ghosh and S. Poddar, Compressed supersymmetry at $14 \mathrm{TeV}$ LHC, Phys. Rev. D 89 (2014) 037702 [arXiv:1308.1526] [InSPIRE].

[33] Particle Data Group collaboration, J. Beringer et al., Review of Particle Physics (RPP), Phys. Rev. D 86 (2012) 010001 [inSPIRE].

[34] Aleph Collaboration, Delphi Collaboration, l3 Collaboration, OPAL Collaboration, LeP Working Group for Higgs Boson Searches collaboration, S. Schael et al., Search for neutral MSSM Higgs bosons at LEP, Eur. Phys. J. C 47 (2006) 547 [hep-ex/0602042] [INSPIRE].

[35] U. Ellwanger, C.-C. Jean-Louis and A.M. Teixeira, Phenomenology of the General NMSSM with Gauge Mediated Supersymmetry Breaking, JHEP 05 (2008) 044 [arXiv: 0803.2962] [INSPIRE]. 
[36] U. Ellwanger, J.F. Gunion and C. Hugonie, NMHDECAY: A Fortran code for the Higgs masses, couplings and decay widths in the NMSSM, JHEP 02 (2005) 066 [hep-ph/0406215] [INSPIRE].

[37] U. Ellwanger and C. Hugonie, NMHDECAY 2.0: An Updated program for sparticle masses, Higgs masses, couplings and decay widths in the NMSSM, Comput. Phys. Commun. 175 (2006) 290 [hep-ph/0508022] [INSPIRE].

[38] M. Badziak, M. Olechowski and S. Pokorski, New Regions in the NMSSM with a $125 \mathrm{GeV}$ Higgs, JHEP 06 (2013) 043 [arXiv: 1304.5437] [INSPIRE].

[39] F. Domingo and U. Ellwanger, Updated Constraints from B Physics on the MSSM and the NMSSM, JHEP 12 (2007) 090 [arXiv:0710.3714] [INSPIRE].

[40] A. Crivellin, J. Rosiek, P.H. Chankowski, A. Dedes, S. Jaeger et al., SUSY_FLAVOR v2: A Computational tool for FCNC and CP-violating processes in the MSSM, Comput. Phys. Commun. 184 (2013) 1004 [arXiv: 1203. 5023] [INSPIRE].

[41] A. Crivellin and J. Rosiek, SUSY_FLAVOR library for rare decays in the MSSM, PoS (EPS-HEP 2013) 081 [arXiv: 1308.6299] [INSPIRE].

[42] G. Bélanger, F. Boudjema, A. Pukhov and A. Semenov, MicrOMEGAs 3 : A program for calculating dark matter observables, Comput. Phys. Commun. 185 (2014) 960 [arXiv: 1305.0237] [INSPIRE].

[43] WMAP collaboration, G. Hinshaw et al., Nine-Year Wilkinson Microwave Anisotropy Probe (WMAP) Observations: Cosmological Parameter Results, Astrophys. J. Suppl. 208 (2013) 19 [arXiv: 1212.5226] [INSPIRE].

[44] Planck collaboration, P.A.R. Ade et al., Planck 2013 results. XVI. Cosmological parameters, Astron. Astrophys. (2014) [arXiv:1303.5076] [INSPIRE].

[45] J. Alwall, M. Herquet, F. Maltoni, O. Mattelaer and T. Stelzer, MadGraph 5 : Going Beyond, JHEP 06 (2011) 128 [arXiv:1106.0522] [INSPIRE].

[46] T. Sjöstrand, S. Mrenna and P.Z. Skands, PYTHIA 6.4 Physics and Manual, JHEP 05 (2006) 026 [hep-ph/0603175] [INSPIRE].

[47] W. Beenakker, R. Hopker, M. Spira and P.M. Zerwas, Squark and gluino production at hadron colliders, Nucl. Phys. B 492 (1997) 51 [hep-ph/9610490] [INSPIRE].

[48] W. Beenakker, R. Hopker and M. Spira, PROSPINO: A Program for the production of supersymmetric particles in next-to-leading order QCD, hep-ph/9611232 [INSPIRE].

[49] W. Beenakker, S. Brensing, M. Krämer, A. Kulesza, E. Laenen et al., Squark and Gluino Hadroproduction, Int. J. Mod. Phys. A 26 (2011) 2637 [arXiv:1105.1110] [InSPIRE].

[50] M. Krämer, A. Kulesza, R. van der Leeuw, M. Mangano, S. Padhi et al., Supersymmetry production cross sections in pp collisions at $\sqrt{s}=7 \mathrm{TeV}$, arXiv:1206.2892 [INSPIRE].

[51] M. Drees, H. Dreiner, D. Schmeier, J. Tattersall and J.S. Kim, CheckMATE: Confronting your Favourite New Physics Model with LHC Data, arXiv:1312.2591 [INSPIRE].

[52] DELPHES 3 collaboration, J. de Favereau et al., DELPHES 3, A modular framework for fast simulation of a generic collider experiment, JHEP 02 (2014) 057 [arXiv:1307.6346] [INSPIRE].

[53] E. Conte, B. Fuks and G. Serret, MadAnalysis 5, A User-Friendly Framework for Collider Phenomenology, Comput. Phys. Commun. 184 (2013) 222 [arXiv:1206.1599] [INSPIRE]. 
[54] E. Conte and B. Fuks, MadAnalysis 5: status and new developments, J. Phys. Conf. Ser. 523 (2014) 012032 [arXiv: 1309.7831] [INSPIRE].

[55] ATLAS collaboration, A search for resonant Higgs-pair production in the $b \bar{b} b \bar{b}$ final state in pp collisions at $\sqrt{s}=8 \mathrm{TeV}$, ATLAS-CONF-2014-005.

[56] CMS Collaboration, Search for resonant HH production in 2gamma+2b channel, CMS-PAS-HIG-13-032.

[57] ATLAS collaboration, Search for Higgs Boson Pair Production in the $\gamma \gamma b \bar{b}$ Final State using pp Collision Data at $\sqrt{s}=8 \mathrm{TeV}$ from the ATLAS Detector, arXiv:1406.5053 [INSPIRE].

[58] CMS Collaboration, Search for supersymmetry in hadronic final states using MT2 with the CMS detector at $\sqrt{s}=8 \mathrm{TeV}$, CMS-PAS-SUS-13-019.

[59] ATLAS collaboration, Search for massive particles in multijet signatures with the ATLAS detector in $\sqrt{s}=8 \mathrm{TeV}$ pp collisions at the LHC, ATLAS-CONF-2013-091.

[60] CMS Collaboration, Search for light- and heavy-flavor three-jet resonances in multijet final states at $8 \mathrm{TeV}$, CMS-PAS-EXO-12-049.

[61] CMS collaboration, Search for pair-produced dijet resonances in four-jet final states in $p p$ collisions at $\sqrt{s}=7 \mathrm{TeV}$, Phys. Rev. Lett. 110 (2013) 141802 [arXiv:1302.0531] [INSPIRE].

[62] CMS collaboration, Search for microscopic black holes in pp collisions at $\sqrt{s}=8 \mathrm{TeV}$, JHEP 07 (2013) 178 [arXiv: 1303.5338] [INSPIRE].

[63] A. Datta, A. Djouadi, M. Guchait and F. Moortgat, Detection of MSSM Higgs bosons from supersymmetric particle cascade decays at the LHC, Nucl. Phys. B 681 (2004) 31 [hep-ph/0303095] [INSPIRE].

[64] P. Bandyopadhyay, A. Datta and B. Mukhopadhyaya, Signatures of gaugino mass non-universality in cascade Higgs production at the LHC, Phys. Lett. B 670 (2008) 5 [arXiv:0806.2367] [INSPIRE].

[65] K. Huitu, R. Kinnunen, J. Laamanen, S. Lehti, S. Roy et al., Search for Higgs Bosons in SUSY Cascades in CMS and Dark Matter with Non-universal Gaugino Masses, Eur. Phys. J. C 58 (2008) 591 [arXiv:0808.3094] [INSPIRE].

[66] P. Bandyopadhyay, Probing non-universal gaugino masses via Higgs boson production under SUSY cascades at the LHC: A Detailed study, JHEP 07 (2009) 102 [arXiv:0811.2537] [INSPIRE].

[67] S. Gori, P. Schwaller and C.E.M. Wagner, Search for Higgs Bosons in SUSY Cascade Decays and Neutralino Dark Matter, Phys. Rev. D 83 (2011) 115022 [arXiv:1103.4138] [InSPIRE].

[68] G.D. Kribs, A. Martin, T.S. Roy and M. Spannowsky, Discovering the Higgs Boson in New Physics Events using Jet Substructure, Phys. Rev. D 81 (2010) 111501 [arXiv:0912.4731] [INSPIRE].

[69] G.D. Kribs, A. Martin, T.S. Roy and M. Spannowsky, Discovering Higgs Bosons of the MSSM using Jet Substructure, Phys. Rev. D 82 (2010) 095012 [arXiv:1006.1656] [INSPIRE].

[70] A. Belyaev, J.P. Hall, S.F. King and P. Svantesson, Novel gluino cascade decays in $E_{6}$ inspired models, Phys. Rev. D 86 (2012) 031702 [arXiv:1203.2495] [inSPIRE].

[71] B. Bhattacherjee, A. Chakraborty, D. Kumar Ghosh and S. Raychaudhuri, Using Jet Substructure at the LHC to Search for the Light Higgs Bosons of the CP-Violating MSSM, Phys. Rev. D 86 (2012) 075012 [arXiv:1204.3369] [INSPIRE]. 
[72] A. Belyaev, J.P. Hall, S.F. King and P. Svantesson, Discovering $E_{6} S U S Y$ models in gluino cascade decays at the LHC, Phys. Rev. D 87 (2013) 035019 [arXiv:1211.1962] [InSPIRE].

[73] M. Cacciari, G.P. Salam and G. Soyez, FastJet User Manual, Eur. Phys. J. C 72 (2012) 1896 [arXiv: 1111.6097] [INSPIRE].

[74] M. Cacciari, G.P. Salam and G. Soyez, The Anti-k(t) jet clustering algorithm, JHEP 04 (2008) 063 [arXiv:0802.1189] [INSPIRE].

[75] M.J. Dolan, C. Englert and M. Spannowsky, Higgs self-coupling measurements at the LHC, JHEP 10 (2012) 112 [arXiv: 1206.5001] [InSPIRE].

[76] A. Papaefstathiou, L.L. Yang and J. Zurita, Higgs boson pair production at the LHC in the $b \bar{b} W^{+} W^{-}$channel, Phys. Rev. D 87 (2013) 011301 [arXiv:1209.1489] [inSPIRE].

[77] A.J. Barr, M.J. Dolan, C. Englert and M. Spannowsky, Di-Higgs final states augMT2ed selecting hh events at the high luminosity LHC, Phys. Lett. B 728 (2014) 308 [arXiv: 1309.6318] [INSPIRE].

[78] V. Barger, L.L. Everett, C.B. Jackson and G. Shaughnessy, Higgs-Pair Production and Measurement of the Triscalar Coupling at LHC(8,14), Phys. Lett. B 728 (2014) 433 [arXiv: 1311.2931] [INSPIRE].

[79] D.E. Ferreira de Lima, A. Papaefstathiou and M. Spannowsky, Standard model Higgs boson pair production in the $(b \bar{b})(b \bar{b})$ final state, JHEP 08 (2014) 030 [arXiv: 1404.7139] [INSPIRE]. 